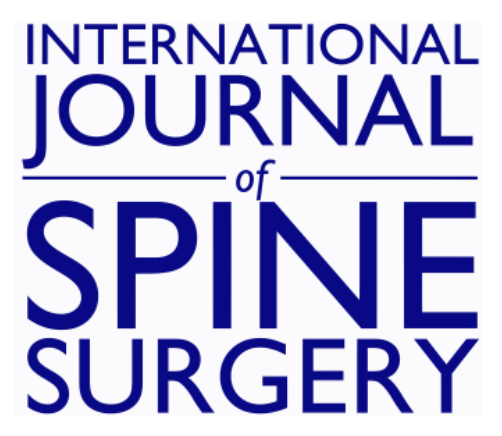

\title{
Transforaminal Endoscopic Decompression of the Lumbar Spine for Stable Isthmic Spondylolisthesis as the Least Invasive Surgical Treatment Using the YESS Surgery Technique
}

ANTHONY YEUNG and VIT KOTHEERANURAK

Int J Spine Surg 2018, 12 (3) 408-414

doi: https://doi.org/10.14444/5048

http://ijssurgery.com/content/12/3/408

This information is current as of April 26, 2023.

Email Alerts Receive free email-alerts when new articles cite this article. Sign up at:

http://ijssurgery.com/alerts

The International Journal of Spine Surgery

2397 Waterbury Circle, Suite 1,

Aurora, IL 60504, Phone: +1-630-375-1432 


\title{
Transforaminal Endoscopic Decompression of the Lumbar Spine for Stable Isthmic Spondylolisthesis as the Least Invasive Surgical Treatment Using the YESS Surgery Technique
}

\author{
ANTHONY YEUNG, MD, ${ }^{1}$ VIT KOTHEERANURAK, MD ${ }^{2}$ \\ ${ }^{I}$ Neurosurgery Department, University of New Mexico School of Medicine, Albuquerque, New Mexico and Desert Institute for Spine Care, Phoenix, Arizona, \\ ${ }^{2}$ Spine Unit, Orthopedic Department, Queen Savang Vadhana Memorial Hospital, Sri Racha, Chonburi, Thailand
}

\begin{abstract}
The first author's series has reported and published his 5- to 10-year results in a preliminary review of endoscopic transforaminal decompression for degenerative and isthmic spondylolisthesis causing sciatica and back pain. This study was initiated due to favorable results in relieving both back and leg pain after a spine surgeon with isthmic spondylolisthesis who wanted to avoid fusion requested consideration for an endoscopic procedure for his own isthmic spondylolisthesis condition. After listening to the first author's podium presentation on selective endoscopic discectomy and foraminal decompression under local anesthesia in 1995, he requested consideration for transforaminal endoscopic decompression for recent onset of progressive sciatica from his isthmic spondylolisthesis. He was the first patient to undergo endoscopic decompression in our series of 55 patients from January 2002 to December 2012 that served as the database for a clinical case series review of selective endoscopic discectomy and foraminoplasty in patients with degenerative spondylolisthesis who specifically chose to stage the author's endoscopic transforaminal decompressive trademarked procedure over fusion.

The patients were evaluated and considered for the endoscopic procedure specifically at their request, even though they were informed about the traditional "gold standard" fusion for surgical intervention. At that time the medical field did not have the database to study outcomes other than the favorable clinical results found in patients who underwent selective endoscopic discectomy and foraminoplasty for degenerative spondylolisthesis. These patients were accepted for endoscopic spine surgery in a shared clinical decision after full disclosure. The patients opted specifically for the Yeung Endoscopic Spine Surgery (YESS) technique that included foraminoplasty and dorsal endoscopic rhizotomy from the author's evolving clinical experience of 10,000 cases over 27 years.
\end{abstract}

Endoscopic Minimally Invasive Surgery

\section{INTRODUCTION}

This article is on highly vetted and stratified indications for isthmic spondylolisthesis only, using an evolving YESS technique of foraminal decompression and dorsal ramus rhizotomy treating the pain generators in the lumbar spine for both back pain and sciatica in lieu of traditional open decompression and instrumented fusion. Patients who elected to forgo open translaminar decompression and/or fusion as a traditional option provided the initial case series study. Their choice was to continue with nonsurgical care or undergo traditional spine surgery. Isthmic spondylolisthesis, diagnosed by magnetic resonance imaging and computed tomography scan included those patients who had concomitant patho-anatomy such as disc protrusion, herniation, and spinal stenosis. These patients were offered the endoscopic transforaminal decompressive procedure if they received significant relief with diagnostic and therapeutic steroid blocks. ${ }^{1,2}$ The patients also had spine consultations that opined that, after translaminar decompression, a fusion was the accepted surgical protocol. These patients had to be satisfied with the results of the diagnostic and therapeutic injections before they were offered endoscopic surgery. They were told that their decision to have endoscopic surgery first may have to be staged should their condition progress. They could then decide to go on to fusion in the future. The endoscopic procedure would not interfere with a fusion as a secondary procedure. Patients who did not respond favorably to their satisfaction were advised to either continue with nonsurgical treatment 
or to seek fusion as the definitive procedure. None went on to fusion.

It was found that the YESS endoscopic transforaminal decompression technique as a stand-alone or staged procedure may reduce the number of surgical fusions in the face of both degenerative and isthmic spondylolisthesis, since the YESS technique demonstrated favorable results by targeting the pain generator. In the author's clinical experience, when transforaminal decompression and dorsal endoscopic ablation of the branches of the dorsal ramus were added to the endoscopic procedure to address back pain as well as sciatica, even better clinical outcomes were obtained. Endoscopic decompression did not further destabilize the spine. ${ }^{3}$

Chronic low back pain will afflict most people in their lifetime due to disc degeneration from trauma or from the normal aging process. Recent clinical studies from the University of Hong Kong demonstrate that imaging studies detecting disc degeneration even in asymptomatic patients will eventually result in the development of low back pain severe enough for patients to seek medical and surgical treatment. Degenerative conditions in an aging spine are presented in cadaver cryosections by Wolfgang Rauschning. Pfirrmann also describes a degenerative cascade that matches the Rauschning's cryosections with corresponding magnetic resonance images. Chronic low back pain is a close third, only after number 1, diabetes, and essentially tied with ischemic heart disease, number 2 , in the top 5 common health condition expenditures according to an analysis published by JAMA: The Journal of the American Medical Association in 2013.

\section{METHOD}

This clinical research article provides evidence that treating the pain generator is a viable option for isthmic spondylolisthesis in selected patients.

The transforaminal endoscopic technique by one of us (A.Y.) trademarked as the Yeung endoscopic technique (YESS) is utilized for isthmic spondylolisthesis in selected patients. The YESS technique is differentiated from the more generic percutaneous endoscopic discectomy technique known as percutaneous endoscopic lumbar discectomy (PELD), marketed by competing endoscopic spine companies by other endoscopic spine colleagues and key opinion leaders.

The YESS technique differs due to its intradiscal "inside-out" philosophical emphasis on intradiscal therapy, and dorsal endoscopic rhizotomy in addition to the "outside-in" foraminal decompression technique. Both techniques target the pathoanatomy and pain generators. It was also discovered that visualizing the nerves, ligaments, and osteophytes in the foramen provides surgical opportunities to relieve pain by endoscopic decompression of these pain-generating structures. The endoscopic visualization of foraminal nerves and ligaments also match microanatomy that is unfamiliar to traditional surgeons.

Trajectories of other key opinion leaders vary in their methods of targeting the patho-anatomy. These key opinion leaders mostly emphasize serial dilation, a time-honored method of other endoscopic techniques to safely decompress bone or soft tissue to reach the targeted pathology.

The purpose of this communication is to not only to describe the surgical method, but to also to demonstrate the endoscopic treatment of the visualized patho-anatomy aided by endoscopic probing under local anesthesia. All patients were satisfied with their decision to avoid fusion for their condition that resulted in both back pain and sciatica. In these patients, sciatica was more of a bother than debilitating pain. Most were aware of their pars defect as a result of a developmental defect, acquired defect, trauma, or other unknown causes.

\section{DISCUSSION}

Degenerative spondylolisthesis following translaminar decompression for spinal stenosis is recognized as a possible sequela of surgical open translaminar decompression. This has prompted more careful selection of patients who require decompression but may be able to avoid fusion.

Spondylolisthesis is a spinal condition for which, 10 to 15 years ago, fusion was accepted as a "gold standard" for surgical stabilization.

The rationale that spondylolisthesis was a result of instability, a time-justified rationale further evaluated by flexion/extension imaging, has created a surgical thought process that fusion for demonstrated instability should be a part of any primary or revision surgery. Fusion surgery was then augmented and supplanted by various dynamic stabilization techniques ranging from pedicle-based versus interspinous-based dynamic stabilization.

With over 20 years of experience treating patients who sought endoscopic decompression in order to avoid the "dreaded" fusion recommended by spine 
Life-table method of survival analysis: Degenerative and isthmic spondylolisthesis following SED and transforaminal endoscopic decompression $(\mathrm{N}=55)$

18-mo reprieve
From fusion
2 yr $64 \%$ will
avoid fusion
5 yr $33 \%$ will
avoid fusion

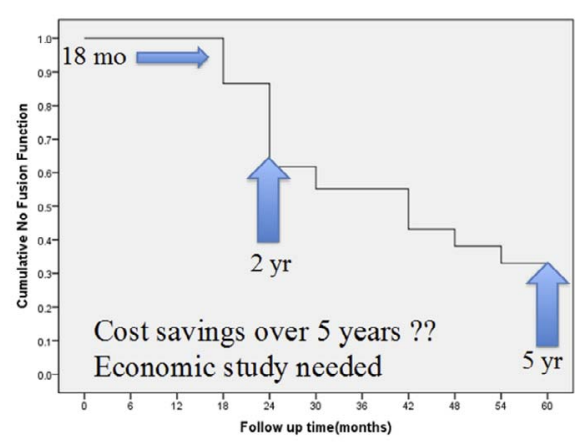

SED = Selective endoscopic decompression

Figure 1. Life-table method of survival analysis: degenerative and isthmic spondylolisthesis following selective endoscopic discectomy and foraminal decompression.

surgeons, coupled with knowledge of patients, friends, and other physicians recommending fusion only as a "last resort," the author reviewed his personal database of patients who sought him out for endoscopic decompression and who refused fusion by opting for endoscopic decompression first. This resulted in the publication of a series of opinionbased level 4 and 5 evidence-based medicine articles by Yeung in open access journals dating back to December 2015.

Yeung's first article, "Moving Away From Fusion," was published on December 2, 2015, in the Journal of Spine. A review of 58 patients with degenerative and isthmic spondylolisthesis from 2003 to 2013 was studied. No statistical analysis was performed or powered for this study, but a careful review of the results from the review created a stratification strategy based on the best outcomes and feedback from Yeung's returning patients who maintained contact or who returned with minor symptoms in follow-up visits. These patients were specifically instructed to notify Yeung if their spinal condition worsened after discharge. Since Yeung was the only endoscopic spine surgeon available and was initially sought out by these patients, most remained in close contact if their symptoms progressed to the point that they would consider additional surgery. The patients' satisfaction with the index surgery universally expressed in follow-up visits stimulated Yeung's interest in following his patients' experiences. There were 58 patients with 10 years of follow-up. The patients included highperformance athletes and spine surgeons who maintained contact after they sought the less invasive endoscopic decompression procedure over fusion, as the "gold standard" mainly for degenerative spondylolisthesis at the time. Some of the patients were featured on the author's practice website when the patients volunteered to be featured as surgical case reports or offered testimonials. Some of their surgeries were featured on playlists on the author's website and placed on YouTube.

From Yeung's initial review of the 58 patients with a 5- to 10-year follow-up, the lifetime analysis found that approximately $36 \%$ avoided additional surgery but $100 \%$ were satisfied with their decision to delay fusion as their first surgical option (Figure 1).

As the author closely followed these patients, he continued stratification of patient selection from his evolving endoscopic surgery technique, aided by new instrumentation and decompressive techniques that surgically facilitated foraminal decompression utilizing his "inside-out" intradiscal therapy philosophy and transforaminal decompression methods. From these clinical data the author then identified those patients most likely to benefit from endoscopic transforaminal decompression, even with isthmic spondylolisthesis, by first performing transforaminal diagnostic and therapeutic injections to identify the pain segment and using a criteria of $70 \%$ improvement as a sign that transforaminal decompression would provide longer pain relief if the patient was very satisfied with the injection results. The clinical endoscopic decompression outcome was more favorable if they had weeks or months of lasting relief. Patients with isthmic spondylolisthesis were more reluctantly offered foraminal decompression when their main complaint was not sciatica, but had predominant back pain. The diagnostic and therapeutic injections helped define patients who could expect at least reasonable intermediate-term pain relief results when the patients chose endoscopic decompression first, as a "staged procedure." Adding medial branch blocks to the transforaminal therapeutic injections provided even better results.

Patients that had more advanced conditions that would benefit better with fusion were referred to the traditionally fellowship-trained spine surgeons in Yeung's group, Desert Institute of Spine Care, to be considered for fusion, or back to referring spine surgeons for fusion unless they insisted on trying Yeung's staged procedure first. Many elderly patients over 65 were more likely to want a staged procedure when their pain more recently affected their lifestyle or quality of life. All were satisfied 
Table 1. Two-year follow-up outcomes following transforaminal endoscopic decompression for degenerative spondylolisthesis.

\begin{tabular}{lccc}
\hline & Preoperative & Postoperative & $\boldsymbol{P}$ value \\
\hline Oswestry Disability Index score & $27.1 \pm 9$ & $17.6 \pm 10$ & $<.001$ \\
Visual analogue scale score & $5.0 \pm 2.8$ & $2.2 \pm 1.6$ & $<.001$ \\
Patients who opted for fusion & & $4 / 32(12.5 \%)$ & \\
$\quad$ as a staged procedure & & & \\
\hline
\end{tabular}

with their original decision to avoid fusion, even if their back pain was still present, but decreased enough that they were satisfied to no longer curtail their physical activity level.

A 2-year follow-up database from 32 stratified patients with degenerative spondylolisthesis that underwent transforaminal endoscopic decompression from 2012 to 2105 at the author's institute showed significantly good outcomes with regard to the Oswestry Disability Index and Visual Analogue Scale scores. Only $4 / 32(12.5 \%)$ patients chose to have fusion after 2 years. Fusion following transforaminal endoscopic decompression for the patients with degenerative spondylolisthesis was therefore significantly reduced (Tables 1 and 2).

The series of the patients with low-grade isthmic spondylolisthesis that underwent the transforaminal endoscopic decompression from 2012 to 2016 demonstrated good to excellent outcomes. Moreover, none of them opted for any further fusion (mean follow-up time, 41.6 months).

\section{The Future}

Fusion is generally acknowledged as the surgical treatment for symptomatic spondylolisthesis. Multiple minimally invasive fusion implants and techniques have emerged in the past 10 years, creating an explosion of minimally invasive surgical procedures touting minimal invasiveness. Current emphasis is now also on attaining sagittal alignment and balance, another phase in the evolution of fusion techniques. Now, some key opinion leaders among spine surgeons are recognizing and acknowledging that not all, or even most, patients require instrumented

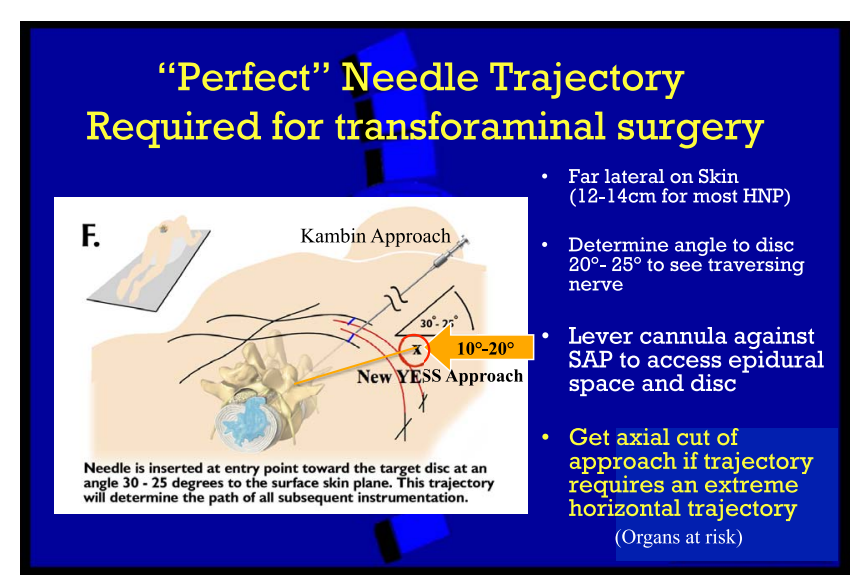

Figure 2. Evolving trajectories to access the patho-anatomy. The YESS approach targeting the patho-anatomy in isthmic spondylolisthesis.

fusion. Endoscopic decompression, whether translaminar or transforaminal, (the least invasive) will eventually lead the way for the staging of procedures that offer the patient more treatment choices. Of the minimally invasive techniques, the transforaminal decompressive approach is the least invasive.

Degenerative spondylolisthesis following translaminar decompression for spinal stenosis is recognized as a possible sequela of surgical translaminar decompression. This has prompted more careful selection of patients who require decompression but may be able to avoid fusion.

A recent randomized, controlled trial published in the New England Journal of Medicine of patients presenting with lumbar spinal stenosis, with or without degenerative spondylolisthesis, concluded that adding a fusion to decompression surgery did not result in better clinical outcomes at 2 years and 5 years. Instead, it significantly increased direct hospital costs, including the costs of surgery and the in-hospital stay.

A recent 2-year follow-up case report in Neurosurgical Focus 2016 on "Transforaminal Endoscopic Discectomy to Relieve Sciatica and Also Delay Fusion in a 31-Year Old Man With Pars Defect and Low Grade Spondylolisthesis" by the Department of Neurological Surgery at the University of

Table 2. Outcomes of patients following transforaminal endoscopic decompression for isthmic spondylolisthesis (2012 to 2016 ).

\begin{tabular}{|c|c|c|c|c|c|c|}
\hline No. & Age, y & Level of Operation & ODI, pre/post & VAS, pre/post & $\begin{array}{c}\text { Modified } \\
\text { Macnab's Criteria }\end{array}$ & $\begin{array}{l}\text { Outcome/Duration } \\
\text { From Surgery, mo }\end{array}$ \\
\hline 1 & 62 & $\mathrm{~L} 5 / \mathrm{S} 1$ & $48 / 32$ & $7 / 2$ & Excellent & Fusion-free/64 \\
\hline 2 & 27 & L5/S1 & $32 / 19$ & $7 / 4$ & Good & Fusion-free/61 \\
\hline 3 & 65 & $\mathrm{~L} 5 / \mathrm{S} 1$ & $42 / 28$ & $4 / 1$ & Good & Fusion-free/32 \\
\hline 4 & 71 & $\mathrm{~L} 5 / \mathrm{S} 1$ & $24 / 14$ & $6 / 2$ & Excellent & Fusion-free/26 \\
\hline 5 & 58 & $\mathrm{~L} 5 / \mathrm{S} 1$ & $17 / 12$ & $4 / 0$ & Excellent & Fusion-free/25 \\
\hline
\end{tabular}

Abbreviations: ODI, Oswestry Disability Index; VAS, visual analogue scale; pre/post, preoperative/postoperative. 


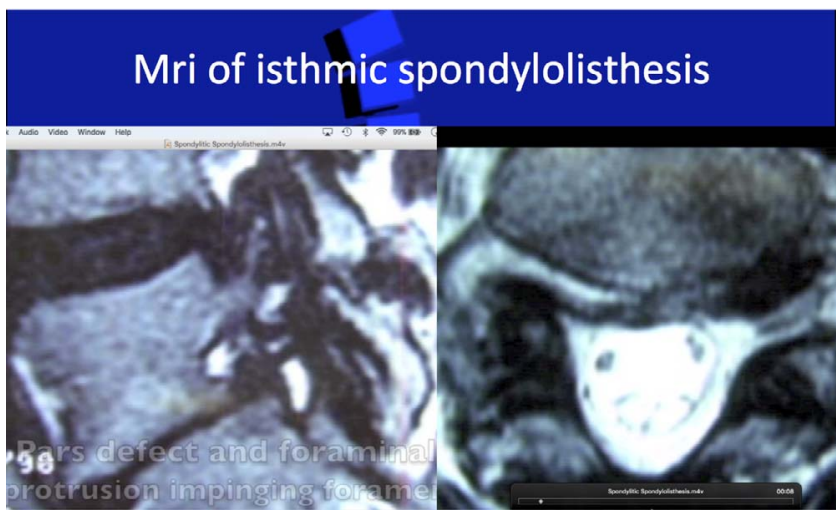

Figure 3. Symptoms: sciatica $>$ back pain.

Washington and the University of Miami Miller School of Medicine supports the findings of this article with 5-year follow-up that the preservation of critical structures through the transforaminal endoscopic approach and avoidance of traditional translaminar open destabilizing approaches is an important surgical concept that needs further study.

Needle, cannula, and instrument trajectories have also evolved. Ideal trajectories were noted to shorten the surgical procedure and provide better clinical results since incomplete decompression was a significant known cause of failed back surgery syndrome, especially in the early learning curve. Therefore, an ideal trajectory or "perfect" trajectory was desired. Figure 2 illustrates the evolving trajectories for different targeted patho-anatomic pain sources. It is also cautioned that endoscopic surgeons should not consider the procedure to be a "see one, do one, teach one" procedure. It take time to develop the surgical skills to target and confirm the pathoanatomy endoscopically. New endoscopic systems,

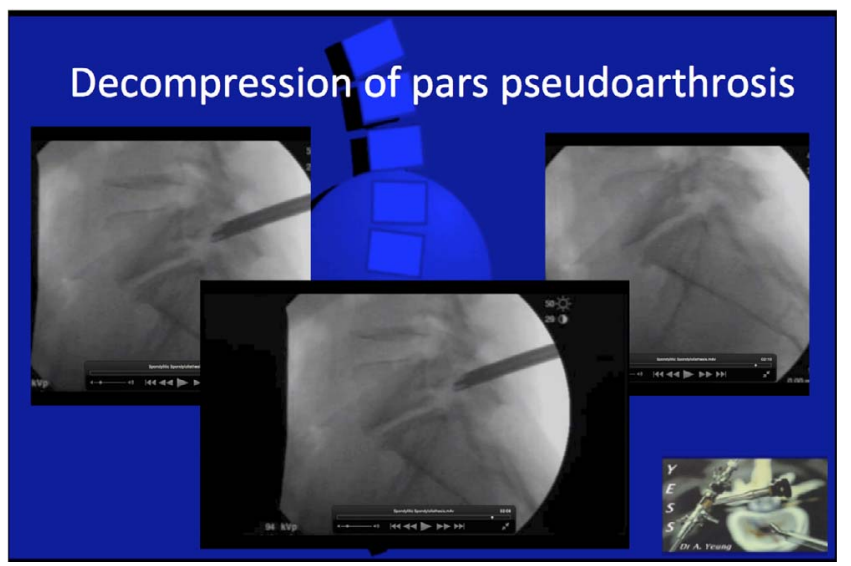

Figure 4. Mechanical decompression at L5-S1. Trephines inside the walls of the cannula, trephines, kerrisons, and lasers are important endoscopic tools to facilitate surgery.

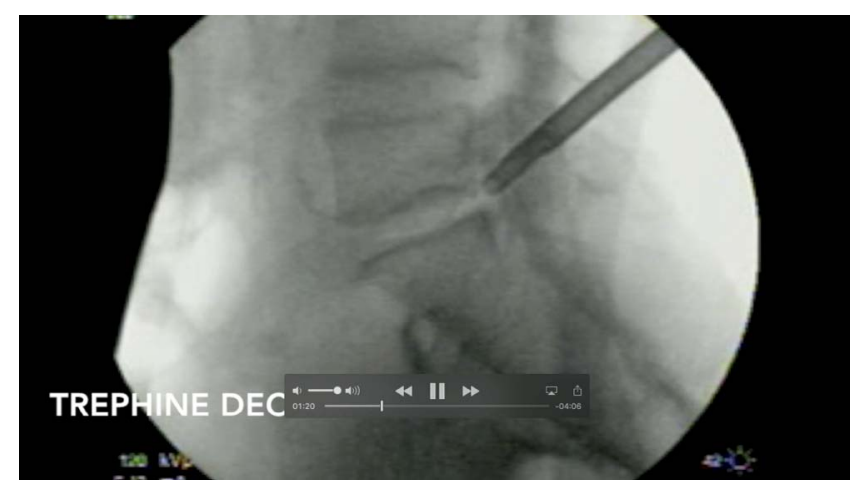

Figure 5. Trephine of the pars pseudo arthrosis impinging on the exiting nerve causing sciatica in a young person with isthmic spondylosis. Sciatica worsens with narrowing from disc degeneration and aging, demonstrating disc narrowing and spondylolisthesis.

surgical tools, and surgical experience may be needed to reproduce the best results.

\section{Health Care Reform}

With the cost of health care, and spine care specifically, contributing to the escalation and affordability of health care expenditures, payment by singlepayer systems and insurance payers is under duress. Each stakeholder should consider the rationale and cost of health care delivery with a consideration toward necessity and affordability. In some patients, degeneration resulted in deterioration to a grade 1 to grade 2 spondylolisthesis from aging or postoperative open decompression not present preoperatively.

\section{Lessons Learned by Foraminal Endoscopic} Decompression and Endoscopic Rhizolysis in the Past 27 Years

(1) The horizontal sagittal alignment of the facet at the level of the index surgery was helpful in predicting further slippage following endoscopic decompression.

(2) Endoscopic transforaminal decompression did not cause additional slippage.

(3) Patients with a favorable response to transforaminal epidural blocks performed by an experienced endoscopic surgeon predicted a favorable response to transforaminal endoscopic decompression.

(4) Foraminal endoscopic decompression did not result in further slippage, especially if the sagittal alignment of the facet was more horizontal than vertical.

(5) Patients over age 65 who were laborers and who had retired from their day jobs respond- 


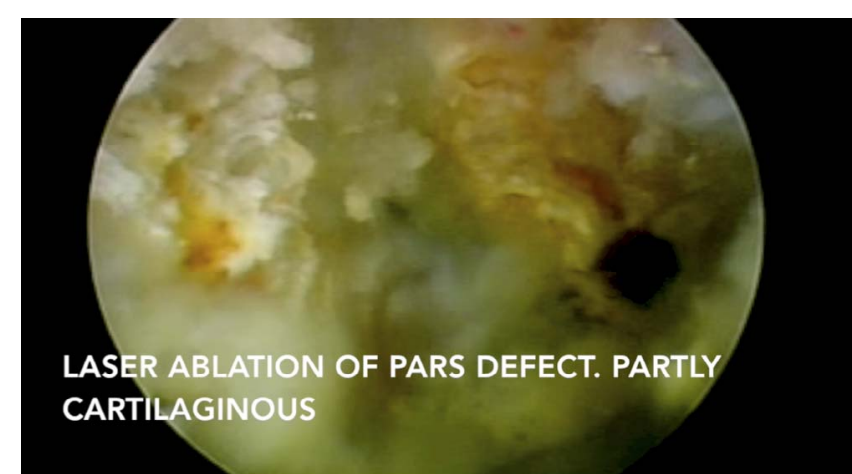

Figure 6. Endoscopic visualization of the decompressed pars pseudoarthrosis impinging on the exiting nerve. Back pain in tall discs with pars defects are well tolerated until aging causes symptomatic lumbar spondylosis, treatable with subsequent dorsal rhizotomy as a staged separate procedure or as a hybrid procedure.

ed better than younger active patients who were still working, but none regretted having their surgery staged.

(6) If back pain represented $30 \%$ to $50 \%$ of their pain complaint, a hybrid procedure that included dorsal endoscopic rhizotomy relieved back pain as well.

The treatment algorithm in spinal care is evolving and changing due to a better understanding of the patho-anatomy, well correlated with its pathophysiology. The author has written about his own evolving technique in publications including a series of articles in Surgical Technology International. The author has decided to review his results of isthmic spondylolisthesis, excluding degenerative spondylo-

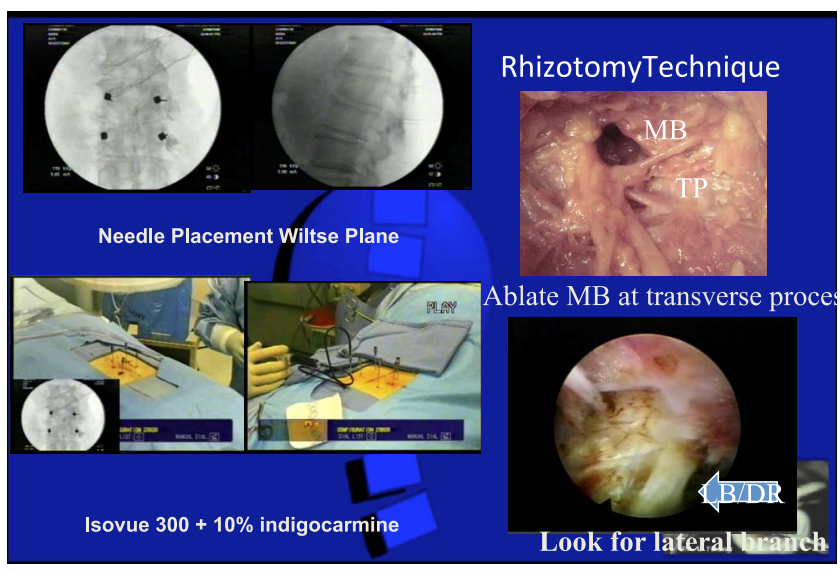

Figure 7. Endoscopic visualized rhizotomy. The branches of the dorsal ramus innervating the facet joints can be found in the foramen ventral to the intertransverse ligament during foraminoplasty as well as crossing the transverse process during dorsal endoscopic rhizotomy. A hybrid procedure to ablate the branches of the dorsal ramus provides the best surgical treatment for facet pain. listhesis with 10-year follow-up. The results mirror the results of a less-stratified procedure.

\section{CONCLUSION}

This small series illustrates that the majority of patients should consider the "gold standard" instrumented fusion that produces very good surgical results. However, there is a small group of patients with recent onset of back pain and sciatica who may benefit from transforaminal decompression and nerve ablation.

The following images illustrate case examples of Isthmic spondylolisthesis (Figures 3 through 7).

\section{REFERENCES}

1. Yeung AT. Endoscopic decompression for degenerative and isthmic spondylolisthesis. J Neurol Disord. 2017;5(6):371. https://doi.org/10.4172/2329-6985.1000371

2. Yeung AT, Kotheeranurak V. Transforaminal endoscopic decompression of the lumbar spine for stable degenerative spondylolisthesis as the least invasive surgical treatment using the YESS surgery technique. J Spine. 2018;7(1):407. https://doi.org/10.4172/2165-7939.1000407

3. Yeung AT. Transforaminal endoscopic decompression for painful degenerative conditions of the lumbar spine: a review of one surgeon's experience with over 10,000 cases since 1991. J Spine Neurosug. 2017;6(3). https://doi.org/10.4172/ 2325-9701.1000266

\section{ADDITIONAL REFERENCES}

Yeung AT, Gore S. In-vivo endoscopic visualization of patho-anatomy in symptomatic degenerative conditions of the lumbar spine II: intradiscal, foraminal, and central canal decompression. Surg Technol Int. 2011;XXI:299-319.

Yeung AT, Gore S. Twenty-three years of experience with percutaneous transforaminal spine surgery: its evolution, the painful conditions treated, results, personal thoughts, and a review of the evolving literature. Surg Technol Int. XXV.

Yeung AT. Moving away from fusion by treating the pain generator: the secrets of an endoscopic master. $J$ Spine. 2015;4:e121.

Yeung AT. Endoscopic decompression, foraminalplasty and dorsal rhizotomy for foraminal stenosis and lumbar spondylosis: a hybrid procedure in lieu of fusion. J Neurol Disord. 2016;4:322.

Yeung AT. Endoscopic decompression for degenerative and isthmic spondylolisthesis. J Spinal Disord. 2017;5(6):371.

Försth P, Ólafsson G, Carlsson T, et al. A randomized, controlled trial of fusion surgery for lumbar spinal stenosis. $N$ Engl J Med. 2016;374:1413-1423.

Yeung AT, Gore S. Endoscopically guided foraminal and dorsal rhizotomy for chronic axial back pain based on cadaver and endoscopically visualized anatomic study. Int J Spine Surg. 2014;8:23.

Yeung AT. Robotics in the MIS spine surgery arena: a new 
role to advance the adoption of endoscopic surgery as the least invasive spine surgery procedure. J Spine. 2017;6:374.

Yeung AT. Delivery of spine care under health care reform in the United States. $J$ Spine. 2017;6:372.

Madhavan K, Chieng LO, Hofstetter CP, Wang MY. Transforaminal endoscopic discectomy to relieve sciatica and delay fusion in a 31-year-old man with pars defects and lowgrade spondylolisthesis Neurosurg Focus. 2016;40(2):E4.
Published 15 August 2018

This manuscript is generously published free of charge by ISASS, the International Society for the Advancement of Spine Surgery. Copyright (c) 2018 ISASS. To see more or order reprints or permissions, see http://ijssurgery.com. 